\title{
Gene Deletion Abnormality
}

National Cancer Institute

\section{Source}

National Cancer Institute. Gene Deletion Abnormality. NCI Thesaurus. Code C45583.

Loss of a DNA sequence that contains one or more genes. This typically occurs via rearrangement, whereby DNA sequences that are normally separated become joined together. A gene deletion abnormality can be either heritable or somatic. 